\title{
PREVALENCIA DE CARIES DE LA INFANCIA TEMPRANA Y FACTORES DE RIESGO ASOCIADOS EN NIÑOS DE 1 A 5 AÑOS EN LA CIUDAD DE SANTIAGO DE CALI
}

\author{
${ }^{1}$ María Cristina Arango De La Cruz, ${ }^{2}$ Adriana Jaramillo Echeverry, ${ }^{3}$ Carlos Arturo Cruz Valderrama \\ ${ }^{1}$ Odontólogo U. del Valle, Especialista en Odontología Pediátrica U. de Valle, Candidata a Magíster en Epidemiología U. del Valle, \\ Docente Escuela de Odontología U. del Valle, Colombia. \\ ${ }^{2}$ Odontólogo U. del Valle, Magíster en Microbiología U. de Valle, Magíster en Epidemiología U. del Valle, Docente Escuela de Odontología U. del Valle, Colombia. \\ ${ }^{3}$ Licenciado de Matemáticas U. del Valle, Docente Escuela de Odontología U. del Valle, Colombia.
}

Autor responsable de correspondencia: María Cristina Arango De La Cruz Correo electrónico: titiarango25@yahoo.com

\section{RESUMEN}

Objetivo: determinar la prevalencia de Caries de la Infancia Temprana (CIT) en niños de 1 a 5 años de la ciudad de Cali y describir los factores de riesgo asociados.

Materiales y métodos: se realizó un estudio descriptivo transversal, de prevalencia con todas las instituciones educativas inscritas en la Secretaría de Educación. La muestra la constituyeron 929 menores a quienes se les indagó mediante un cuestionario dirigido a sus padres, acerca de los factores de riesgo asociados con caries dental. Se realizó un examen clínico intraoral para determinar el índice ceo-d según los criterios de la Organización Mundial de la Salud y se evaluó el índice de placa bacteriana de Sillness y Löe. Se realizó un análisis univariado para describir las variables y conocer la distribución de la variable de salida. Para el bivariado se utilizó la prueba $\mathrm{Chi}^{2}$ y la correlación de Spearman, según el tipo de variable. Para comparación del índice ceo-d según estrato socioeconómico y hábitos de higiene como el cepillado antes de acostarse, se utilizó la prueba de Kruskal-Wallis.

Resultados: se encontró una prevalencia de CIT de $29,3 \%$, siendo esta mayor en el estrato socioeconómico bajo. De los factores de riesgo analizados, se encontró una asociación significativa con no cepillar al niño antes de dormir con un OR de 1,50 [IC 95\%: 1,09 - 2,07, p= $0,008]$.

Conclusiones: la prevalencia de caries dental fue mayor que la reportada para Cali en el ENSAB III a los 5 años. Se utilizó como índice para caries dental el ceo-d sin incluir lesiones precavitacionales, lo que podría subestimar la prevalencia de esta entidad en la población de estudio. [Arango MC, Jaramillo A, Cruz CA. Prevalencia de caries de la infancia temprana y factores de riesgo asociados en niños de 1 a 5 años en la ciudad de Santiago de Cali. Ustasalud 2013; 12: 108 - 115]

Palabras clave: Caries dental, Placa dental, Prevalencia, Preescolar.

\section{PREVALENCE OF EARLY CHILDHOOD CARIES AND ASSOCIATED RISK FACTORS AMONG 1-5 YEAR OLD PRE-SCHOOLERS OF SANTIAGO DE CALI}

\section{ABSTRACT}

Objective: to assess the prevalence of Early Childhood Caries (ECC) among 1-5 year old pre-schoolers in the city of Cali and describe the associated risk factors.

Methods: a descriptive study of prevalence in all educational institutions registered in the Ministry of Education was carried out. The sample consisted of 929 children who were asked by a questionnaire sent to their parents about the risk factors associated with dental caries. An intraoral examination was performed to determine the dmf-t index according to the criteria of the World Health Organization and the plaque index of Sillness and Löe was also evaluated. Univariate analysis was performed to describe the variables and know the distribution of the dependent variable. For the bivariate analysis, the $\mathrm{Chi}^{2}$ test and Spearman correlation was used depending on the type of variable. For comparison of the dmf-t according to socioeconomic and hygiene conditions such as brushing before bed, the Kruskal-Wallis test was used.

Results: a prevalence of $29.3 \%$ of ECC was found, this value was greater in the lower socioeconomic condition. Of the risk factors analyzed, we found a significant association with the absence of brushing before going to bed with an OR of 1.50 [IC $95 \%$ : $1.09-2.07$, $\mathrm{p}=0.008)$

Conclusions: the prevalence of dental caries was higher than that reported for Cali in the ENSAB III at 5 years. The caries index was dmf-t that excludes precavitacional lesions, which could be underestimating the real prevalence of dental caries in the study population.

Key words: Dental caries, Dental plaque index, Prevalence, Infants.

Recibido para publicación: noviembre 20 de 2013. Aceptado para publicación: 13 de diciembre de 2013. 


\section{INTRODUCCIÓN}

La Caries de la Infancia Temprana (CIT) es definida como la presencia de una o más lesiones cariosas (cavitadas o no), dientes ausentes debido a caries dental u obturados en cualquier diente deciduo en niños menores de 71 meses. ${ }^{1-4}$ Hoy en día, esta entidad se considera un problema de salud pública que afecta a los infantes en todo el mundo. ${ }^{5}$ Anteriormente era conocida como "caries del biberón", asociada con el hábito de dar tetero a la hora de dormir, pero en los últimos años se ha determinado que no existe una relación directa entre este hábito y la aparición de la enfermedad, pues estudios concluyeron que muchos niños que dormían con el tetero, no desarrollaron caries dental. Algunos autores sugieren que más del $12 \%$ de los niños en edad preescolar están afectados, aunque en ciertas poblaciones la afección es tan alta que alcanza un $70 \% .^{6-9}$ Los niños con esta entidad tienden a mantener un riesgo alto de sufrir caries dental en la dentición permanente. ${ }^{10}$

La salud oral de los niños se encuentra muy relacionada con el periodo gestacional de la madre, ya que las alteraciones que se presentan durante este periodo pueden afectar la salud buco dental del hijo, es evidente la importancia de que el personal médico tenga los conocimientos necesarios, para establecer programas preventivos, además de detectar las enfermedades orales más frecuentes y de esta manera orientar y remitir al paciente donde el odontólogo.?

Los riesgos para presentar CIT se ven incrementados cuando la presencia de placa bacteriana es excesiva o las técnicas de higiene bucal son inadecuadas, ${ }^{11-13}$ siendo la zona del contorno gingival la más involucrada con la retención de placa bacteriana. ${ }^{10}$ Otro indicador importante es la presencia de placa bacteriana en la región de incisivos superiores, que produce la desmineralización y destrucción de los tejidos dentarios debido a la acción enzimática de las bacterias presentes en la cavidad bucal, donde el tiempo de exposición a la enfermedad y la actitud individual de los padres en torno al problema constituyen variables de importancia.

El uso de jarabes para controlar la tos y las afecciones gripales son factores relevantes en el desarrollo de la caries de la infancia temprana, ya que contienen hasta un $70 \%$ de sacarosa y, en algunas ocasiones, las madres abusan de ellos durante la noche puesto que su contenido de antihistamínicos induce al sueño. El consumo elevado e indiscriminado de azúcares durante el día, es otro aspecto predisponente para que inicie el proceso de desmineralización de las superficies dentarias. Otros factores relacionados son la exposición frecuente a carbohidratos de alimentos que no se proporcionan en el tetero, sumado a la presencia de hipoplasias en el esmalte. Estudios recientes han sugerido la relación entre la caries de la infancia temprana y el bajo peso al nacer, las complicaciones en la etapa fetal o las dificultades en el parto debido a la predisposición de estos niños a presentar defectos en el esmalte o a la posibilidad de que sean niños cronicamente enfermos reconfortados mediante biberones azucarados. ${ }^{12}$

Algunos factores de riesgo son considerados como asociados y entre ellos se cuentan los sociales, como son el nivel socioeconómico, así como el nivel educativo y cultural de la familia. Weinstein y Riedy (2001), demostraron la alta correlación entre el bajo nivel educativo de la madre y presencia de caries de la infancia temprana. ${ }^{11}$ En un estudio realizado por la Universidad del Bosque (Colombia), se determinó que el nivel educativo de la madre y el entorno socioeconómico de la familia eran las variables de mayor asociación con la presencia de caries de la infancia temprana. ${ }^{14}$ Otros aspectos que aumentan el riesgo de desarrollar la enfermedad están asociados con el hecho de pertenecer a una familia inmigrante y en los niños de mayor edad el consumo de golosinas y bebidas azucaradas. Otros indicadores son bajo peso al nacer, hermanos con caries de la infancia temprana, baja exposición a los fluoruros y escasa higiene oral. Es importante hallar las comunidades en que se concentran este tipo de factores, para realizar programas preventivos y educativos.

Para el desarrollo de lesiones cariosas y más de tipo generalizado como lo es la CIT, el papel de los microorganismos, los carbohidratos fermentables y las alteraciones estructurales de los dientes, sumado a una susceptibilidad marcada del huésped son importantes, interactuando como un conjunto, al ser el principal factor la presencia de placa bacteriana. Algunos autores sugieren que el componente más importante para el desarrollo de caries dental no es la presencia de azúcares, sino el tiempo de exposición a los alimentos. ${ }^{1-3}$

El principal microorganismo implicado en la colonización temprana es Streptococcus mutans al ser uno de los principales agentes responsables de la caries dental por su habilidad de adhesión a los dientes. ${ }^{5,13}$ Sin embargo, intervienen numerosos factores tales como: la resistencia del esmalte a la disolución ácida, la frecuencia y los hábitos higiénicos, y la influencia de la saliva con sus enzimas antibacterianas. ${ }^{16}$ La colonización del $S$. mutans se da después de la erupción del primer diente temporal, ya que requiere de una superficie como la dentaria para su 
adherencia, colonización y posterior degradación de los minerales y generalmente, ocurre como resultado de una transmisión vertical, es decir, de madre a hijo. ${ }^{12}$

La transmisión también puede ser de tipo horizontal, como lo reportaron Alaluusua y Renkonen, quienes realizaron un estudio con niños de un jardín entre 2 y 4 años y pudieron comprobar que las colonias de $S$. mutans en sus cavidades bucales eran muy similares, lo que podía indicar una posible transmisión entre los niños. ${ }^{15}$

La enfermedad es caracterizada por la presencia de lesiones en superficies lisas, con mayor frecuencia en los incisivos superiores y en los primeros molares deciduos; este patrón de enfermedad es importante debido al progreso rápido y destructivo. Se observan lesiones de mancha blanca desde que los incisivos aparecen en la boca, si no se realiza una intervención temprana en poco tiempo evolucionarán a lesiones cavitadas localizadas en el tercio gingival, aunque también puede presentarse en las superficies proximales expuestas. Los caninos y los segundos molares pueden afectarse a medida que erupcionan. ${ }^{1}$ El tratamiento a tan temprana edad se dificulta por la poca colaboración del paciente por lo que el objetivo principal es inactivar el proceso y posponer la fase de restauración definitiva hasta que el niño tolere los procedimientos clínicos aunque esto no es posible en todos los casos por la agresividad de las lesiones; por esta razón en determinados casos es necesario realizar el tratamiento bajo anestesia general. ${ }^{6,18}$ Para inactivar el proceso es necesaria la cooperación de los padres, quienes deben recibir instrucción y motivación.

Es importante conocer la prevalencia de la caries de la infancia temprana y realizar así un diagnóstico precoz con el fin de definir e instaurar programas de prevención específicos en esta población y que su impacto sea efectivo, si se tiene en cuenta que esta entidad se ha convertido en un problema de salud pública que afecta a niños menores de 71 meses ( 5 años 11 meses), que es una de las principales causas de morbilidad oral y que no existen datos exactos sobre su prevalencia, por lo menos en la ciudad de Cali. Además de la inquietud generada en pediatras y odontopediatras debido al gran número de casos de caries dental en esta población y siendo su manejo tan complicado,

De este modo se evita tener que desarrollar programas terapéuticos costosos puesto que los datos epidemiológicos demuestran que la efectividad del control de caries de la infancia temprana, se basa en la prevención y no en el tratamiento restaurativo. Se ha sugerido que una dificultad relacionada con el control de la caries de la infancia temprana es que pocos padres llevan a los niños a consulta odontológica antes de los tres años y si lo hacen, es debido a una urgencia o a un problema específico y no por control.

Aunque la caries dental es una enfermedad que presenta factores determinantes y predisponentes, no se ha logrado establecer un acuerdo entre el sector gubernamental y los profesionales de la salud oral sobre cuál factor juega el papel principal en su aparición. Por otro lado, se piensa que este tipo de enfermedades están limitadas a poblaciones de estratos socioeconómicos bajos o personas con niveles educativos mínimos. De acuerdo con estos antecedentes, el objetivo de la presente investigación fue determinar la prevalencia de Caries de la Infancia Temprana en la población de 1 a 5 años de edad en la ciudad de Cali, en el 2006, e identificar los factores de riesgo que propicien el desarrollo de caries dental en la población infantil de estudio.

\section{MATERIALES Y MÉTODOS}

Se realizó un estudio descriptivo transversal, de prevalencia. La población incluyó niños entre 1 y 5 años que asistieron a centros de educación preescolar (hogares infantiles, jardines, guarderías) de la ciudad del Cali, en el 2006.

\section{Muestreo}

Se realizó un muestreo por conglomerados al utilizar como unidad muestral las instituciones de preescolar. Se estratificó la población de instituciones en tres estratos mediante la variable estrato socioeconómico y teniendo en cuenta que la caries dental es una entidad heterogénea (en términos de su varianza) en estos subgrupos. Para disminuir esta heterogeneidad se utilizó el muestreo probabilístico estratificado. Con un ajuste de la muestra de un $10 \%$ calculado a partir de una prevalencia esperada de caries dental del 50\%, el tamaño final fue 1164 niños distribuidos proporcionalmente según el peso de cada estrato. El margen de error para el total de la muestra fue de \pm $4,0 \%$ para un nivel de confianza del $90 \%$. Durante el procedimiento de muestreo se seleccionaron las unidades primarias (instituciones) de forma aleatoria proporcional para cada estrato (bajo, medio, alto). Debido a la difícil accesibilidad a todos los sitios y lo complejo de contar con el permiso de los padres, se logró evaluar en promedio diez niños por institución.

\section{Criterios de selección}

Para la recolección de la muestra se tomó como criterio de exclusión niños con enfermedad sistémica, síndromes o cualquier tipo de discapacidad motora o sensorial. Se contó con el consentimiento informado de los padres así como con el asentimiento del menor 
según lo avalado por el Comité de Ética Institucional de la Universidad del Valle (CIREH) y de acuerdo con la Resolución No. 008430 del Ministerio de Salud de Colombia del 4 de octubre de 1993.

\section{Recolección de información}

A cada padre se le entregó un cuestionario para indagar sobre factores de riesgo social y biológico. Para el examen clínico se utilizó una unidad portátil con lámpara y un asiento con luz directa e instrumental estéril para diagnóstico. A cada paciente se le tomó el índice de placa bacteriana según Silness y Löe, posteriormente se limpiaron las superficies con gasa o cepillo de dientes asegurando una superficie limpia para el diagnóstico de caries dental, el cual fue evaluado mediante el índice ceo-d, y así calcular la prevalencia de caries dental de acuerdo con los parámetros de la Organización Mundial de la Salud. El diagnóstico clínico fue realizado por dos operadores previamente calibrados con un nivel de acuerdo de 0,8 y una persona auxiliar quien se encargó del registro de los datos. Al finalizar el examen se realizó una charla a los niños y a las madres comunitarias o profesores sobre higiene oral.

\section{Análisis estadístico}

Posterior a la recolección de los datos, se realizó la construcción de la base de datos con doble digitación a través del programa Microsoft Excel 2010. Para el análisis estadístico se utilizó el programa estadístico Stata 11.0. El nivel de significancia estadística fue establecido con una $\mathrm{p}<0,05$.

Se hizo un análisis exploratorio para determinar el comportamiento de la variable resultado ceo-d usando la prueba de Kolmogorov-Smirnov (D: 0,67 $\mathrm{p}=0,000$ ) en la que se observó una distribución no normal. Durante el análisis exploratorio se encontró una mayor proporción de niños en estratos bajos al ser este un factor de riesgo para caries dental, se estratificó y se buscaron diferencias dentro de los estratos por medio de la prueba de KruskalWallis.

El análisis univariado incluyó la distribución de caries dental y variables sociodemográficas, de conocimiento y hábitos, estimando intervalos de confianza del $95 \%$. La variable resultado (caries dental) se analizó tanto de manera categórica (presencia) ausencia) como numérica (índice ceo-d). Se realizó un análisis bivariado por cada una de las variables "factores de riesgo" a partir de la presencia de caries dental utilizando la prueba Chi cuadrado para identificar la independencia entre las variables categóricas y la correlación de Spearman para las variables numéricas.Para determinar diferencias en el índice ceo-d según estrato socioeconómico y hábitosde higiene como el cepillado antes de acostarse, se utilizó la prueba de Kruskal-Wallis.

\section{RESULTADOS}

La muestra del estudio estuvo constituida por 929 niños, distribuidos por sexo en una relación 1:1. La prevalencia de caries dental, es decir, la proporción de niños que presentaron un índice de ceo-d mayor de 1 , fue de $29,28 \%$ [IC 95\%: 26,35 - 32,21].

La edad promedio de los participantes fue 3,58 años, con una desviación estándar de 1,07. El índice de dientes temporales afectados por caries (ceo-d) fue de 1,33 en promedio, con una desviación estándar de 2,90. En la Tabla 1 se presenta la descripción de la población de estudio, según las variables sociodemográficas.

Al comparar el promedio de edad entre los niños con caries y sin caries, en el primer grupo fue de 3,69 años [IC 95\%: 3,58 - 3,80] y en el segundo de 3,54 [IC 95\%: 3,46 - 3,63], con un rango intercuartílico de 1 y una mediana de 4 para ambos grupos. En la Tabla 2 se observa la distribución de la población según la presencia o no de caries dental.

El $27,1 \%$ de las niñas y el $31,5 \%$ de los niños presentaron caries dental sin encontrarse diferencias significativas por sexo (Chi²: $2,14 \mathrm{p}=0,143$ ). Con respecto a las variables sociodemográficas comparadas con la presencia o ausencia de la caries dental, no se encontraron diferencias por sexo, nivel educativo de los padres, tipo de familia o número de hijos. Según la distribución de la presencia o ausencia de caries dental por estrato socioeconómico, se encontró una diferencia significativa entre tipo de servicios de salud y estratos socioeconómicos ( $\left.\mathrm{Chi}^{2}: 16,88 \mathrm{p}=0,005\right)$. Para evaluar esta diferencia por estratos, se tomó el índice ceo-d por cada uno y se realizó la prueba Kruskal Wallis (KW: 6,9 $\mathrm{p}=0,003$ ) encontrándose que al menos dos grupos socioeconómicos mostraron una diferencia estadísticamente significativa en el ceo-d, por lo que se realizó la prueba de Bonferroni que permitió determinar que la diferencia estaba entre los estratos alto y bajo $(p=0,033)$

Se contrastó además el índice ceo-d, con el cepillado después del tetero y el no cepillarse antes de acostarse y se observaron diferencias entre los grupos solamente con los que no se cepillaban antes de acostarse (KW: 7,43 $\mathrm{p}=0,006)$.

Con respecto a las variables consideradas como factores de riesgo (Tabla 3) se encontró una relación significante solo al acostarse sin cepillarse, con un OR de 1,50 [IC 95\%: 1,09 - 2,07 p=0,008). Al analizar la correlación entre el índice ceo-d y el índice de placa bacteriana, se evidenció un coeficiente de Spearman de 0,46 (p<0,005). 
Tabla 1. Distribución de la población según variables sociodemográficas.

\begin{tabular}{cc}
\hline Variable & $\mathrm{n}(\%)$ \\
\hline
\end{tabular}

\section{Caries dental}

No

$657(70,72)$

Sí

$272(29,28)$

Sexo

Femenino

$465(50,05)$

Masculino

$464(49,95)$

Estrato

Bajo

$512(55,11)$

Medio

$368(39,61)$

Alto

$49(5,27)$

Nivel educativo del padre o madre

$\begin{array}{lc}\text { Ninguno } & 12(1,30) \\ \text { Primaria } & 115(12,50) \\ \text { Secundaria } & 453(49,20) \\ \text { Tecnológico } & 149(16,20) \\ \text { Universitario } & 139(15,10) \\ \text { Posgrado } & 38(4,10) \\ \text { Otro } & 14(1,50)\end{array}$

Tipo de familia

Nuclear

$504(54,80)$

Extensa

$244(26,50)$

Compuesta

$23(2,50)$

Múltiple

$22(2,40)$

Dividida

$127(13,80)$

Número de hijos en la familia

Uno

$370(40,26)$

Dos

$358(38,96)$

Tres o más

$191(20,78)$

Servicio de salud

Ninguno

$193(20,78)$

Sisben

$181(19,48)$

EPS, otro

$555(59,74)$

\section{DISCUSIÓN}

La prevalencia de CIT es diferente entre países en todo el mundo con valores que varían desde el $4 \%$ hasta el $90 \%$, convirtiéndose en un problema de salud pública. Esta prevalencia varía según el desarrollo de los países, así como en estratos dentro de los mismos. Los resultados de la prevalencia de este estudio $(29,8 \%)$ son más altos que los reportados en países desarrollados como Estados Unidos o Canadá en donde oscila entre $4 \%$ y $56 \% 9$ mayor a lo reportado por el Tercer Estudio Nacional de Morbilidad Bucal (ENSAB III) para Cali $(24,1 \%)$ y menor a lo sugerido a nivel de Colombia lo cual fue de $54,8 \%$, con una historia de caries dental a los 5 años de $60,4 \% .^{17}$

Con respecto al ceo-d, el ENSAB III ${ }^{17}$ reportó un valor de 3 a los 5 años para Colombia y un valor de 2,1 para Cali siendo este mayor al observado en este estudio $(1,33)$. Al tener en cuenta estos hallazgos y que el ENSAB III utilizó población mayor de 5 años, mientras que en este trabajo se evaluó la población de 1 a 5 años es evidente que se requiere instaurar programas de prevención para evitar su aumento. Con respecto al sexo no se encontraron diferencias como si lo reportaron Díaz-Cárdenas y González-Martínez. ${ }^{19}$

Para el ceo-d los estratos socioeconómicos con mayor proporción de caries dental fueron el bajo (1 y 2) con $32,2 \%$ y el medio ( 3 y 4 ) con $26,1 \%$, lo que coincide con lo observado por schamschula y colaboradores quienes manifestaron que el desarrollo económico y la condición socioeconómica son factores fuertemente relacionados con la etiología de la caries dental puesto que los niños de estrato socioeconómico alto tienen mayor posibilidad de realizar su higiene bucal con pasta de dientes con flúor y de forma frecuente..$^{20}$

Con respecto al cepillado, los hallazgos de este estudio coinciden con lo reportado por un estudio en Taiwán que encontró una fuerte asociación entre la frecuencia del cepillado de los dientes y la prevalencia de caries dental; así mismo observaron que los niños que no se cepillaban los dientes antes de ir a la cama presentaban más lesiones de caries dental. ${ }^{7}$ En cuanto a los valores del índice de placa, hubo una mediana correlación con el índice ceo-d, lo que se explica porque la placa bacteriana es una causa necesaria, más no suficiente para el desarrollo de caries dental y es más importante su composición que su cantidad.

Reisine y Psoter reportaron que factores sociales como la educación de la madre, ingresos, y estrato socioeconómico son determinantes en la salud bucal de los niños, cobrando gran importancia el análisis de los factores sociales en el desarrollo de la caries dental. ${ }^{21}$ En relación con la afiliación al sistema de salud, se ha considerado como riesgo la caries dental, en este estudio se encontró una mayor oportunidad a presentar caries dental 
Tabla 2. Asociación entre variables sociodemográficas y caries dental.

\begin{tabular}{|c|c|c|c|c|c|c|}
\hline \multirow{3}{*}{ Variable } & \multicolumn{4}{|c|}{ Caries dental } & \multirow{3}{*}{$\mathrm{Chi}^{2}$} & \multirow{3}{*}{$\mathrm{p}$} \\
\hline & \multicolumn{2}{|c|}{ No } & \multicolumn{2}{|c|}{$S i$} & & \\
\hline & $\mathbf{n}$ & $\%$ & $\mathbf{n}$ & $\%$ & & \\
\hline \multicolumn{7}{|l|}{ Sexo } \\
\hline Femenino & 339 & 72,9 & 126 & 27,1 & & \\
\hline Masculino & 318 & 68,5 & 146 & 31,5 & 2,14 & 0,143 \\
\hline \multicolumn{7}{|l|}{ Estrato socioeconómico } \\
\hline Bajo & 347 & 67,8 & 165 & 32,2 & & \\
\hline Medio & 272 & 73,9 & 96 & 26,1 & & \\
\hline Alto & 38 & 77,6 & 11 & 22,5 & 16,88 & 0,005 \\
\hline \multicolumn{7}{|c|}{ Nivel educativo del padre o madre } \\
\hline Ninguno & 8 & 66,7 & 4 & 33,3 & & \\
\hline Primaria & 73 & 63,5 & 42 & 36,5 & & \\
\hline Secundaria & 318 & 70,2 & 135 & 29,8 & & \\
\hline Tecnológica & 108 & 72,5 & 41 & 27,5 & & \\
\hline Universitaria & 106 & 76,3 & 33 & 23,7 & & \\
\hline Posgrado & 29 & 76,3 & 9 & 23,7 & & \\
\hline Otro & 15 & 65,2 & 8 & 34,8 & 6,20 & 0,400 \\
\hline \multicolumn{7}{|l|}{ Tipo de familia } \\
\hline Nuclear & 363 & 72,0 & 141 & 28,0 & & \\
\hline Extensa & 163 & 66,8 & 81 & 33,2 & & \\
\hline Compuesta & 17 & 73,9 & 6 & 26,1 & & \\
\hline Múltiple & 11 & 50,0 & 11 & 50,0 & & \\
\hline Dividida & 96 & 75,6 & 31 & 24,4 & & \\
\hline Sin dato & 7 & 77,8 & 2 & 22,2 & 8,33 & 0,080 \\
\hline \multicolumn{7}{|l|}{ Número de hijos en la familia } \\
\hline Uno & 267 & 72,2 & 103 & 27,8 & & \\
\hline Dos & 247 & 69,0 & 111 & 31,0 & & \\
\hline Tres o más & 136 & 71,2 & 55 & 28,8 & 0,90 & 0,635 \\
\hline \multicolumn{7}{|l|}{ Servicio de Salud } \\
\hline Ninguno & 125 & 64,8 & 68 & 35,2 & & \\
\hline Sisben & 116 & 64,1 & 65 & 35,9 & & \\
\hline EPS, otro & 416 & 75,0 & 139 & 25,1 & 11,95 & 0,003 \\
\hline
\end{tabular}


Tabla 3. Distribución de la caries dental, según variables de conocimiento, prácticas y hábitos de higiene.

\begin{tabular}{|c|c|c|c|c|c|c|}
\hline \multirow{3}{*}{ Variable } & \multicolumn{4}{|c|}{ Caries dental } & \multirow{3}{*}{ OR (IC 95\%) } & \multirow{3}{*}{ p } \\
\hline & \multicolumn{2}{|c|}{ No } & \multicolumn{2}{|c|}{$\mathbf{S i}$} & & \\
\hline & $\mathbf{n}$ & $\%$ & $\mathbf{n}$ & $\%$ & & \\
\hline Conocimientos sobre erupción & 487 & 70,99 & 199 & 29,01 & $0,94(0,67-1,33)$ & 0,738 \\
\hline Reconoce importancia del cepillado & 632 & 70,69 & 262 & 29,31 & $1,03(0,16-10,94)$ & 0,966 \\
\hline Asiste a charlas educativas en salud oral & 449 & 70,93 & 184 & 29,07 & $0,93(0,68-1,30)$ & 0,694 \\
\hline Consulta odontológica antes del año & 441 & 70,45 & 185 & 29,55 & $0,96(0,69-1,32)$ & 0,807 \\
\hline Acuesta al niño sin cepillarse & 388 & 67,71 & 185 & 32,29 & $1,50(1,09-2,07)$ & 0,008 \\
\hline Cepilla al niño con crema dental & 626 & 70,50 & 262 & 29,50 & $1,49(0,61-4,14)$ & 0,351 \\
\hline El niño comparte el cepillo & 53 & 74,65 & 18 & 25,35 & $0,81(0,44-1,45)$ & 0,479 \\
\hline Lleva al niño al odontólogo & 437 & 70,14 & 186 & 29,86 & $1,13(0,82-1,56)$ & 0,427 \\
\hline Lleva al niño para revisión odontológica & 421 & 70,64 & 175 & 29,36 & $1,70(0,83-3,39)$ & 0,102 \\
\hline El niño consume medicamentos en jarabe & 622 & 70,68 & 258 & 29,32 & $1,34(0,58-3,49)$ & 0,465 \\
\hline Realiza cepillado después del jarabe & 107 & 73,29 & 39 & 26,71 & $0,86(0,56-1,30)$ & 0,478 \\
\hline El niño recibe lactancia actual & 28 & 65,12 & 15 & 34,88 & $1,31(0,64-2,60)$ & 0,399 \\
\hline El niño toma tetero & 245 & 74,24 & 85 & 25,76 & $0,75(0,55-1,03)$ & 0,069 \\
\hline Realiza cepillado después del tetero & 107 & 75,89 & 34 & 29,11 & $0,73(0,44-1,18)$ & 0,185 \\
\hline El niño toma leche en el tetero & 195 & 73,58 & 70 & 26,42 & $0,82(0,58-1,14)$ & 0,225 \\
\hline El niño toma jugo en el tetero & 72 & 67,92 & 34 & 32,08 & $1,16(0,72-1,82)$ & 0,501 \\
\hline El niño toma panela en el tetero & 46 & 63,89 & 26 & 36,11 & $1,40(0,81-2,37)$ & 0,184 \\
\hline El niño toma Bienestarina en el tetero & 99 & 70,21 & 42 & 29,79 & $1,02(0,67-1,54)$ & 0,885 \\
\hline El niño usa chupo & 23 & 67,65 & 11 & 32,35 & $1,14(0,49-2,48)$ & 0,719 \\
\hline
\end{tabular}

en niños que no tienen ningún tipo de afiliación o estaban al régimen subsidiado. Tal como lo reporta la literatura, ${ }^{19}$ estos resultados se podrían explicar por la falta de acceso a los servicios, por la mala calidad, o por la falta de educación de los padres y llevar los hijos a consulta odontológica a tempranas edades, lo cual en la actualidad es un problema en los servicios de salud.

Según el presente estudio, el tipo de familia con mayor prevalencia de caries dental fue la extensa, así como una menor educación de los cuidadores, este mismo evento se ha encontrado previamente. ${ }^{19}$ Por tal motivo, se sugiere una relación entre la presencia de caries dental con la educación, el nivel socioeconómico y la descomposición familiar. A partir de los hallazgos observados, se recomienda realizar estudios analíticos para definir los factores de riesgo que deban ser intervenidos en la población.

\section{Conclusiones}

La prevalencia de CIT en niños de 1 a 5 años en este estudio $(29,8 \%)$ fue mayor que la reportada para Cali en el ENSAB III para niños de 5 años.
Si bien es cierto que las variables sociodemográficas, de hábitos y de conocimientos no mostraron significancia estadística para explicar la caries dental en esta población, son útiles como indicadores de riesgo por evaluar en futuros estudios.

Solo se encontró asociación estadística entre la presencia de caries dental y el estrato socioeconómico, la afiliación al servicio de salud y el acostar al niño sin realizar cepillado dental.

\section{BIBLIOGRAFÍA}

1. Fejerskov O, Kidd E. The Disease and its Clinical Management Second Edition. Copenhaguen: BlackwellMunksgaard; 2008. p. 4.

2. Ekstrand KR, Rickets DNJ, Kidd EAM. Occlusal caries: pathology, diagnosis and logical management. Dent Update 2001; 28: 380 - 387.

3. Vachirarojpisan T, Shinada K, Kawaguchi Y, Laungwechakan P, Somkote T, Detsomboonrat P. Early childhood caries in children aged 6 -19 months. Community Dent Oral Epidemiol 2004; 32: 133 - 142.

4. Santos AP, Soviero VM. Caries prevalence and risk factors among children aged 0 to 36 months. Pesqui Odontol Bras 2002; 16: 203-208. 
5. Berkowitz RJ. Causes, treatment and prevention of early childhood caries: a microbiologic perspective. J Can Dent Assoc 2003; 69: 304 - 307.

6. Cárdenas D. Fundamentos de Odontología. Odontología Pediátrica. Tercera Edición. Medellín: Corporación para investigación Biológicas; 2003. p. 158.

7. King NM, Wu II, Tsai JS. Caries prevalence and distribution, and oral health habits of zero to four year old children in Macau, China. J Dent Child 2003; 70: 243-249.

8. Cariño KM, Shinada K, Kawaguchi Y. Early childhood caries in northern Philippines. Community Dent Oral Epidemiol 2003; 31: 81- 89.

9. Peressini S, Leake JL, Mayhall JT, Maar M, Trudeau R. Prevalence of early childhood caries among First Nations children, District of Manitoulin, Ontario. Int J Paediatr Dent 2004; 14: 101 -110.

10. Clarke P, Fraser-Lee NJ, Shimono T. Identifying risk factors for predicting caries in school-aged children using dental health information collected at preschool age. ASDC J Dent Child 2001; 68: 373 - 378, 302 - 303.

11. Weinstein P, Riedy C. The reliability and validity of the rapid scale: readiness assessment of parents concerning infant dental decay. ASDC J Dent Child 2001; 68: 129 $135,142$.

12. Tinanoff N, Kanellis MJ, Vargas CM. Current understanding of epidemiology, mechanisms, and prevention of dental caries in preschool children. Pediatric Dent 2002; 24: 543 - 551.

13. Linossier A, Vargas A, Zillmann G, Arrigada M. Streptococci mutans: método semicuantitativo para establecer el rango de riesgo de infección bucal en niños preescolares chilenos. Rev Méd Chile 2003; 131: 412-418.

14. Rey MA, Salas E, Martignon S. Caries dental y asociación a factores de riesgo en la población escolar de Moniquirá, Boyacá. Rev Cient Univ El Bosque 2003; 9: 28 - 34.

15. Alaluusua,Renkonen.Streptococcus mutans establishment and dental caries experience in children from 2 to 4 years old. Scand J Dent Res 1983; 91: 453 - 457.

16. Duque J, Rodríguez A. Factores de riesgo en la predicción de las principales enfermedades bucales en los niños. Rev Cub Estomatol 2001; 38: 111 - 119.

17. Ministerio de Salud y Oficina de Comunicación. República de Colombia. III Estudio Nacional de Salud Bucal (ENSAB III). Tomo VII. Bogotá; 1999. 89 - 90.

18. Tinanoff N, Douglass JM. Clinical decision making for caries management in children. Pediatr Dent 2002; 24: 386 - 392.

19. Diaz-Cardenas S, Gonzalez-Martinez F. Prevalencia de caries dental y factores familiares en niños escolares de Cartagena de Indias. Rev Salud Pública 2010; 12: 843- 851.

20. Schamschula RG, Sugár E, Un PS, Tóth K, Barmes DE, Adkins BL. Physiological indicators of fluoride exposure and utilization: an epidemiological study. Community Dent Oral Epidemiol 1985; 13: 104 - 107.

21. Reisine ST, Psoter W. Socioeconomic status and selected behavioral determinants as risk factors for dental caries. J Dent Educ 2001; 65: 1009 - 1016.

Correos electrónicos de los autores:

María Cristina Arango De La Cruz: titiarango25@yahoo.com Adriana Jaramillo Echeverry: adriana.jaramillo@correounivalle.edu.co Carlos Arturo Cruz Valderrama: cacruzval@yahoo.es

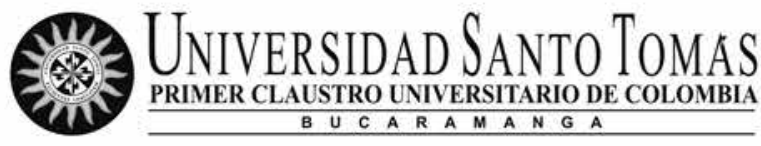

Facultad de

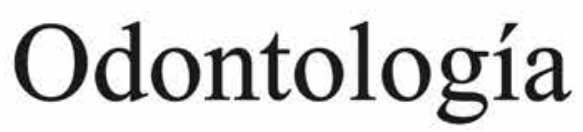

SNIES 1097 - Acreditación de Alta Calidad 517 del 6 de febrero de 2008 - Duración: 10 semestres

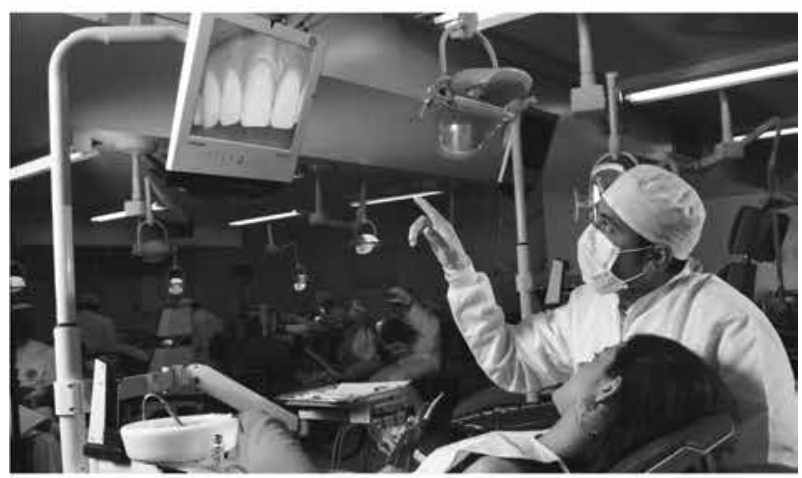

INSCRIPCIONES ABIERTAS

Teléfono: 6800801

ext: 1263 - 1264 - 1265 - 1801

Línea Gratuita 018000917044

promocion@mail.ustabuca.edu.co

BUCARAMANGA

www.ustabuca.edu.co 